\title{
Lipid droplet-associated PAT-proteins show frequent and differential expression in neoplastic steatogenesis
}

\author{
Beate K Straub ${ }^{1}$, Esther Herpel ${ }^{1,2}$, Stephan Singer ${ }^{1}$, Ralf Zimbelmann ${ }^{3}$, Kai Breuhahn ${ }^{1}$, \\ Stephan Macher-Goeppinger ${ }^{1,2}$, Arne Warth ${ }^{1}$, Judith Lehmann-Koch ${ }^{1,2}$, Thomas Longerich ${ }^{1}$, \\ Hans Heid ${ }^{3}$ and Peter Schirmacher ${ }^{1}$ \\ ${ }^{1}$ Department of General Pathology, Institute of Pathology, University Clinic Heidelberg, Heidelberg, Germany; \\ ${ }^{2}$ Tissue Bank of the National Center for Tumor Diseases, University Clinic Heidelberg, Heidelberg, Germany \\ and ${ }^{3}$ Helmholtz Group for Cell Biology, German Cancer Research Center, Heidelberg, Germany
}

\begin{abstract}
In many human cancers, lipogenic pathways are activated; in some tumors, such as hepatocellular carcinoma, this is reflected by the presence of visible lipid droplets. Yet, the biology of steatogenesis in malignant tumors is largely unknown. We have recently shown that lipid droplet-associated proteins of the PAT-family, named after their constituents perilipin (perilipin 1), adipophilin (perilipin 2), and TIP47 (perilipin 3) are differentially expressed in hepatic steatogenesis. We have comprehensively investigated PAT-expression in neoplastic steatogenesis as well as in respective normal tissues with immunohistology and electron microscopy as well as protein biochemical and molecular biological methods. By staining for PAT-proteins, we found lipid droplet accumulation to be a frequent phenomenon of carcinoma cells. Although adipophilin and TIP47 stained almost ubiquitously the rim of lipid droplets in various tumor types, especially those with clear cell phenotype, perilipin was restricted to lipid droplets of hepatocellular adenoma and carcinoma, sebaceous adenoma and carcinoma, and lipomatous tumors. In hepatocellular carcinoma, perilipin, adipophilin, and TIP47 were coexpressed, and showed regional heterogeneity with a predominantly mutually exclusive localization pattern. In step-wise carcinogenesis, adipophilin expression correlated with the proliferation rate and was upregulated during early tumorigenesis, whereas perilipin was often lost during hepatocarcinogenesis. In conclusion, expression analysis of PAT-proteins showed that by far more carcinomas contain (PAT-positive) lipid droplets than expected by conventional light microscopy. PAT-proteins, such as perilipin, are differentially expressed in different tumor types and thus may support diagnostic considerations. Because inhibition of lipogenesis has been shown to exert antineoplastic effects, PAT-proteins may represent targets for interventive strategies. Modern Pathology (2010) 23, 480-492; doi:10.1038/modpathol.2009.191; published online 15 January 2010
\end{abstract}

Keywords: lipid droplet; perilipin; adipophilin; TIP47; hepatocellular carcinoma; tissue microarray

In contrast to normal cells, cancer cells rely mainly on anaerobic glycolysis, a phenomenon termed the Warburg effect. ${ }^{1-2}$ Functionally related, tumor cells show an increased protein, DNA, and fatty acid synthesis. ${ }^{3-4}$ Consistent with this concept, lipogenic pathways are upregulated in diverse human cancers. $^{5-7}$ Some tumors, such as hepatocellular carcinoma (HCC), may show prominent fatty

Correspondence: Dr BK Straub, MD, Institute of Pathology, Im Neuenheimer Feld 220/221, D-69120 Heidelberg, Germany. E-mail: beate.straub@med.uni-heidelberg.de

Received 16 September 2009; revised 2 November 2009; accepted 19 November 2009; published online 15 January 2010 change, as at least some cells in about two-thirds of HCCs are known to contain abundant lipid droplets visible by light microscopy. In particular, early HCC may present with diffuse fatty change. ${ }^{8-9}$ In contrast, other carcinomas usually do not contain visible lipid droplets.

In recent years, knowledge about the structure and organization of lipid droplets has been greatly advanced. ${ }^{10}$ Lipid droplets exist in virtually any kind of normal cell and consist of a triacylglycerol core with a phospholipid monolayer on the surface in which amphiphilic proteins insert. These lipid dropletassociated proteins, such as perilipin, ${ }^{11}$ adipophilin ('adipocyte-differentiation-related protein'), ${ }^{12-13}$ and TIP47 ('tail-interacting protein of $47 \mathrm{kDa}$ '), ${ }^{14}$ subsumed 
as the PAT-family of proteins, and recently redefined as perilipin 1 , perilipin 2 , and perilipin $3,{ }^{15}$ are crucial for the formation, maintenance, modification, and involution of lipid droplets. Gene knockout experiments showed impaired lipid droplet function in certain cellular compartments, such as aberrant adipocyte lipolysis in perilipin-deficient mice $^{16-17}$ and protection against hepatic accumulation of lipid droplets but unimpaired adipogenesis in adipophilin-deficient mice. ${ }^{18}$ Whereas perilipin was thought to be restricted to adipocytes and certain steroidogenic cells, adipophilin and TIP47 are nearly ubiquitously expressed. It is accepted that in adipose tissue peroxisome proliferator-activated receptor- $\gamma$ $(\mathrm{PPAR} \gamma)$ binds to the peroxisome proliferator-response element (PPRE), and activates perilipin gene transcription, whereas in liver, PPAR $\alpha$ binds to this PPRE and thereby the genes of adipophilin and TIP47 are transcribed. ${ }^{19}$ Recently, we showed that this distinction is not absolute and that perilipin becomes de novo expressed in human hepatocytes during steatogenesis. ${ }^{20}$ In chronic liver disease perilipin, adipophilin, and TIP47 show a differential expression pattern regarding cell type, size of lipid droplets, localization in hepatocytes along the portocentral axis, and extent of hepatocellular steatosis. ${ }^{20}$

Neoplastic steatogenesis in diverse carcinomas is triggered by different growth factors, which may induce the nuclear accumulation of transcription factors such as sterol regulatory element binding protein-1 (SREBP-1) through PI3K/Akt pathway thereby leading to upregulation of lipogenic enzymes, such as fatty acid synthase. Interference with this pathway or, eg, fatty acid synthase may preferentially address cancer cells and inhibit tumor growth. ${ }^{21-22,3}$ So far, only a few tumor entities have been analyzed for some PAT-proteins, ${ }^{23-25}$ but more complete analyses are lacking. Owing to their obvious relevance, we have comprehensively investigated the presence and pattern of lipid droplets as well as PAT-expression in human carcinomas.

\section{Materials and methods}

\section{Tissues and Cultured Cells}

All tissue specimens were provided by the tissue bank of the National Center for Tumor Diseases (Heidelberg, Germany; ethics proposal 206/2005, University of Heidelberg). Tumor tissue microarrays of the following organ systems were used: one HCC tissue microarray, one breast cancer tissue microarray, one renal cell carcinoma tissue microarray, one lung cancer microarray, one colorectal carcinoma tissue microarray, and one squamous cell carcinoma of the skin tissue microarray (for details see Tables 1a and b; Supplementary Table 1). Complementary, whole mount sections of seven HCCs, nine hepatocellular adenoma, as well as six focal nodular hyperplasias were analyzed (Table 1b). For the analysis of other tumor entities, a pan-tumor tissue microarray as well as extra tissue sections with altogether 139 paraffin-embedded tissue specimens was used. For the breast cancer, HCC and renal cell carcinoma tissue microarray, each tissue was present in duplicate.

For rtPCR and immunoblot analyses, mRNAs and proteins were isolated from 27 different HCCs and 1 normal human liver sample as described. ${ }^{28}$ For immunofluorescence microscopy and immunoblot of different tumor entities, four cryopreserved human HCCs, one hepatocellular adenoma, one cholangiocarcinoma, one clear cell renal cell carcinoma, one ductal invasive carcinoma of the breast, one adenocarcinoma each of the stomach, colon, prostate gland, pancreas, and lung, as well as one squamous cell carcinoma of the skin and larynx were analyzed. For electron microscopy, tumor specimens including adenocarcinoma of the colon, and stomach, squamous cell carcinoma of the oropharynx, pleomorphic carcinoma and basaloid squamous cell carcinoma of the lung were fixed in glutaraldehyde.

Cultured cells derived from human HCC or human hepatoblastoma were PLC-PRF/5, HepG2, Hep3B, and HuH7. ${ }^{20}$

\section{Antibodies and Reagents}

For primary antibodies used see Supplementary Table 2, for secondary antibodies used see Straub et $a .^{20}$ Nile red (Sigma-Aldrich, St Louis, MO, USA) and Bodipy (Invitrogen, Karlsruhe, Germany) were used to visualize fat.

\section{Immunofluorescence Microscopy}

Immunofluorescence was carried out on cryosections using a lipid-preserving protocol including fixation with $4 \%$ paraformaldehyde or with a conventional protocol including fixation in methanol and acetone as described. ${ }^{20}$ Vectashield Mounting Medium with DAPI (Vector Laboratories, Burlingame, CA, USA) was used. Confocal laserscanning microscopy was performed with a Leica SP5 microscope (Leica, Wetzlar, Germany).

\section{Electron Microscopy}

Tumor specimens $\left(1 \mathrm{~mm}^{3}\right.$ tumor tissue) were fixed in $2.5 \%$ glutaraldehyde and embedded in Epon. Ultrathin sections were prepared for microscopy according to routine procedures. Electron microscopy was performed using a Zeiss analytical EM 902 (Oberkochen, Germany) coupled with a ProScan digital camera (Troendle, Munich, Germany).

\section{Immunohistochemistry, Semiquantitative Evaluation,} and Statistical Analysis

Immunohistochemistry of formalin-fixed, paraffinembedded tissues was performed as described 
Table 1 Immunohistology for PAT-proteins in (a) different tumors and respective normal tissues (excerpt) and (b) hepatocellular tumors and tumor-like lesions

(a) Different tumors and respective normal tissues (excerpt)

\begin{tabular}{|c|c|c|c|c|c|}
\hline Tissue & Tumor/tissue type & $\mathrm{n}$ & $\begin{array}{c}\text { Perilipin } \\
\text { staining (0-3) }\end{array}$ & $\begin{array}{l}\text { Adipophilin } \\
\text { staining (0-3) }\end{array}$ & $\begin{array}{l}\text { TIP47 staining } \\
\qquad(0-3)\end{array}$ \\
\hline \multirow[t]{3}{*}{ Colon } & Tubular adenoma & 25 & 0 & 1.22 & 0.96 \\
\hline & Colorectal adenocarcinoma & 87 & 0 & 1.18 & 1.11 \\
\hline & Normal colon mucosa & 14 & 0 & 0.96 & 0.82 \\
\hline \multirow[t]{4}{*}{ Kidney } & Papillary renal cell carcinoma & 16 & 0 & 1.3 & 0.9 \\
\hline & Clear cell renal cell carcinoma & 69 & 0 & 2.06 & 1.26 \\
\hline & Chromophobic renal cell carcinoma & 17 & 0 & 0.84 & 1.09 \\
\hline & Angiomyolipoma & 19 & 2.55 & 1.08 & 1.0 \\
\hline \multirow[t]{6}{*}{ Liver $^{\mathrm{b}}$} & Cholangiocarcinoma & 5 & 0 & 1.2 & 0.7 \\
\hline & Hepatocellular carcinoma ${ }^{\mathrm{b}}$ & 191 & 0.44 & 1.32 & 0.61 \\
\hline & Dysplastic nodules $^{\mathrm{b}}$ & 9 & 0.5 & 1.5 & 0.5 \\
\hline & Hepatocellular adenomab & 9 & 0.78 & 1.89 & 0.88 \\
\hline & Focal nodular hyperplasia ${ }^{\mathrm{b}}$ & 6 & 1.58 & 1.67 & 0.5 \\
\hline & Nontumorous liver parenchyme ${ }^{\mathrm{b}}$ & 43 & 0.8 & 0.98 & 0.67 \\
\hline \multirow[t]{5}{*}{ Lung } & Squamous cell carcinoma & 68 & 0 & 1.43 & 1.13 \\
\hline & Adenocarcinoma & 63 & 0 & 1.20 & 0.94 \\
\hline & Large cell lung carcinoma & 4 & 0 & 1.5 & 0.75 \\
\hline & Sarcomatoid/pleomorphic lung carcinoma & 4 & 0 & 0.875 & 1.25 \\
\hline & Normal lung parenchyme & 72 & 0 & 1.30 & 0.67 \\
\hline \multirow[t]{5}{*}{ Mammary gland } & Invasive ductal carcinoma & 140 & 0 & 1.21 & 0.92 \\
\hline & Invasive lobular carcinoma & 28 & 0 & 1.29 & 0.95 \\
\hline & Tubular carcinoma & 10 & 0 & 1.05 & 0.9 \\
\hline & Normal mammary gland & 27 & 0 & 1.45 & 0.93 \\
\hline & Mastopathy & 29 & 0 & 1.06 & 0.98 \\
\hline Oropharynx & Squamous cell carcinoma & 6 & 0 & 0.99 & 0.75 \\
\hline Pancreas & Ductal adenocarcinoma & 4 & 0 & 1 & 0.5 \\
\hline Prostate gland & Adenocarcinoma & 4 & 0 & 0.63 & 0.75 \\
\hline \multirow[t]{7}{*}{ Skin } & Squamous cell carcinoma & 62 & $0^{\mathrm{c}}$ & 1.23 & 0.77 \\
\hline & Carcinoma in situ & 18 & 0 & 1.22 & 0.64 \\
\hline & Actinic keratosis & 30 & 0 & 1.12 & 0.85 \\
\hline & Basal cell carcinoma & 6 & 0 & 1.83 & 1.0 \\
\hline & Sebaceous carcinoma & 1 & 2.5 & 2.5 & 2.5 \\
\hline & Sebaceous adenoma & 3 & 1.0 & 2.67 & 1.17 \\
\hline & Normal skin & 6 & $0.5^{\mathrm{d}}$ & 1.42 & 1.2 \\
\hline
\end{tabular}

(b) Hepatocellular tumors and tumor-like lesions

\begin{tabular}{|c|c|c|c|c|c|c|c|c|c|}
\hline \multirow[t]{2}{*}{ Entity } & \multirow{2}{*}{$\begin{array}{l}\text { Tumor grade } \\
\text { (G1-3) }\end{array}$} & \multirow[t]{2}{*}{$\mathrm{n}$} & \multirow{2}{*}{$\begin{array}{l}\text { Mean age } \\
\text { (years) }\end{array}$} & \multirow{2}{*}{$\begin{array}{c}\text { Sex } \\
(\% \text { male })\end{array}$} & \multirow{2}{*}{$\begin{array}{c}\text { Extent of } \\
\text { steatosis (0-3) }\end{array}$} & \multicolumn{2}{|c|}{ Perilipin } & \multirow{2}{*}{$\begin{array}{l}\text { Adipophilin } \\
\text { intens. (0-3) }\end{array}$} & \multirow{2}{*}{$\begin{array}{c}\text { TIP47 } \\
\text { intensity (0-3) }\end{array}$} \\
\hline & & & & & & Intens. (0-3) & Pos. (\%) & & \\
\hline \multirow[t]{4}{*}{ Hepatocellular carcinoma } & All & 191 & 57.1 & 80.5 & 0.20 & 0.44 & 30 & 1.32 & 0.61 \\
\hline & G1 & 18 & 58.6 & 71.4 & 0.33 & 0.31 & 28 & 1.14 & 0.68 \\
\hline & $\mathrm{G} 2$ & 106 & 57.5 & 80.9 & 0.21 & 0.44 & 28 & 1.32 & 0.61 \\
\hline & G3 & 67 & 56.0 & 83.6 & 0.15 & 0.48 & 34 & 1.37 & 0.60 \\
\hline Dysplastic nodules & - & 9 & 55.1 & 85.7 & 0.11 & 0.5 & 44 & 1.28 & 0.5 \\
\hline Hepatocellular adenoma & - & 9 & 34.6 & 11.1 & 0.89 & 0.78 & 66 & 1.89 & 0.88 \\
\hline Focal nodular hyperplasia & - & 6 & 42 & 0 & 0.83 & 1.58 & 100 & 1.67 & 0.5 \\
\hline Nontumorous liver & - & 43 & 55.8 & 50.5 & 0.26 & 0.8 & 70 & 0.98 & 0.67 \\
\hline
\end{tabular}

intens.: intensity; pos.: positivity.

Intensity of immunohistochemical stainings was grouped from 0 (no staining) to 3 (strong staining). ${ }^{26}$

Steatosis was graded from 0 ( $<5 \%$ steatosis) to 3 ( $>66 \%$ steatosis $).{ }^{27}$

${ }^{\mathrm{a}}$ TIP47 staining was often diffusely cytoplasmic and not always seen in a typical lipid droplet pattern.

${ }^{\mathrm{b}}$ See also Table $1 \mathrm{~b}$.

${ }^{\mathrm{C}}$ Nuclear perilipin staining was observed in some squamous cell carcinoma cases.

${ }^{\mathrm{d}}$ Positive perilipin staining was detected in sebaceous glands. For all tumors see Supplementary Table.

previously. ${ }^{20}$ The numbers of perilipin-, adipophilin-, and TIP47-positive lipid droplets were grouped from 0 (no lipid droplets), 1 (few lipid droplets), 2 (moderate number lipid droplets) to 3 (multiple lipid droplets) in analogy to the evaluation of intensity of staining used in the conventional immunoreactivity score system IRS. ${ }^{26}$ The percentage of positive cells was not graded. Fatty change 
observed in nontumorous liver specimens, HCC, dysplastic nodules, hepatocellular adenoma, and focal nodular hyperplasia was scored semiquantitatively in analogy to the scoring system established by Brunt et $a l^{27}$ for steatosis and steatohepatitis, ie, 0 ( $<5 \%$ steatosis), 1 (5-33\%), 2 (34-66\%), and $3(>66 \%)$. Statistical analysis was performed with SPSS version 15.0 for Windows (SPSS, Chicago, IL, USA). Two-sided significance level was $\alpha=$ 0.05. The Mann-Whitney U-test was used to compare patient subgroups for quantitative variables. Spearman's rank correlation was used as a statistical measure of association.

\section{Gel Electrophoresis and Immunoblotting}

Protein isolation from different HCCs and control livers was carried out as described, ${ }^{28}$ protein concentration was measured with the Bradford assay (Bio-Rad, München, Germany), and $20 \mu \mathrm{g}$ protein was used per lane. For other tumors, tissue sections were prepared and directly dissolved in SDScontaining sample buffer. Gel electrophoresis and immunoblotting were performed as described. ${ }^{20}$

\section{PCR Analysis}

In addition to the primers for adipophilin, perilipin, and TIP $47,{ }^{20}$ the following amplification primers were used: actin (forward, 5'-ATGATATCGGCGCGC TCG-3'; reverse, 5'-CGCTCGGTGAGGATCTTCA-3'), PPAR $\alpha$ (forward, 5'-TCTGTCGGGATGTCACAC-3'; reverse, $5^{\prime}$-AGCTCCGTGACGGTCTCC-3'), PPAR $\gamma$ (forward, 5'-CTTGTGAAGGATGCAAGG-3'; reverse, 5'-TCTTTCCTGTCAAGATCG-3').

\section{Results}

\section{Frequent Lipid Droplet Accumulation in Tumorigenesis}

As lipogenic pathways have been described to be upregulated in diverse cancer cells, we analyzed lipid droplet accumulation in different human carcinomas. By confocal laser scanning immunofluorescence microscopy, numerous Nile red- and Bodipy-positive, small lipid droplets were visible in tumor cells of adenocarcinomas and squamous cell carcinoma of different origin (Figure 1a), many more than expected by conventional light microscopy. The number, localization, and size of lipid droplets in different carcinomas varied. Lipid droplets were especially prominent in clear cell renal cell carcinoma (Figure 1a) and HCC (Figure 4). This finding was confirmed by electron microscopy showing small lipid droplets in samples of clear cell renal cell carcinoma, and adenocarcinoma of the colon (Figure 1b), as well as in pleomorphic carcinoma and basaloid squamous cell carcinoma of the lung, and squamous cell carcinoma of the oropharynx.
As expression of the lipid droplet-associated proteins perilipin, adipophilin, and TIP47 has been shown to correlate with lipid droplet accumulation in different tissues and cells, ${ }^{13,20}$ we next investigated PAT-expression in a variety of different human carcinomas. By immunoblot, specific signals for adipophilin and TIP47 were detected in whole-cell lysates of all carcinomas analyzed (Figure 1d). Perilipin protein bands were detected in HCC, but also in whole tissue lysates that contained adipocytes. In immunofluorescence microscopy, adipophilin surrounded Nile red- and Bodipy-positive lipid droplets in carcinoma cells (for clear cell renal cell carcinoma see Figure 1c, for HCC see Figure 4), whereas TIP47 was often localized in the cytoplasm. Perilipin was exclusively detected at the margin of small lipid droplets in HCC (Figure 4), and in adipocytes included in tumor tissue sections. This subcellular localization pattern was confirmed using a pan-tumor array covering all major carcinoma entities (Figures 2 and 3).

In conclusion, lipid droplet accumulation is a frequent feature of carcinoma cells, by far exceeding the extent expected by light microscopy. Immunohistochemical stainings for PAT-proteins mirror the lipid droplet accumulation in normal and neoplastic cells.

\section{Differential PAT-Expression in Carcinogenesis}

To determine frequency and pattern of PAT-expression in different types of carcinomas, we performed immunohistochemical analyses with a large number and different types of human carcinomas ( $n>950$; Table 1; Supplementary Table 1). Adipophilin staining was most prominent in tumors with clear cell phenotype such as clear cell renal cell carcinoma (Figure 2), or clear cell adenocarcinoma of other origin, and in tumors referred to as lipid rich, such as steroid cell tumor of the ovary and apocrine breast cancer. In addition, adipophilin staining was intense in adenocarcinoma of the colon (Figure 2) and in squamous cell carcinoma of different origins. TIP47 surrounded very small lipid droplets, ie in squamous cell carcinoma, cholangiocarcinoma, adenocarcinoma of the colon, and clear cell renal cell carcinoma (Figure 2). Although adipophilin and TIP47-positive lipid droplets were virtually ubiquitous, perilipin was only positive in hepatocellular adenoma and carcinoma, and sebaceous adenoma and carcinoma (Tables 1a and b; Figure 3). In all other epithelial tumors analyzed, and in the respective normal epithelia, no specific perilipin signal was detected (Table 1; Figure 2). For mesenchymal tumors, positive perilipin staining was observed in adipogenic tumors such as lipoma and liposarcoma, hibernoma, as well as angiomyolipoma of the kidney (Figure 3) and in normal uni- and plurivacuolar adipocytes. Whereas most tumor entities were positive only for adipophilin and TIP47, expression of all 

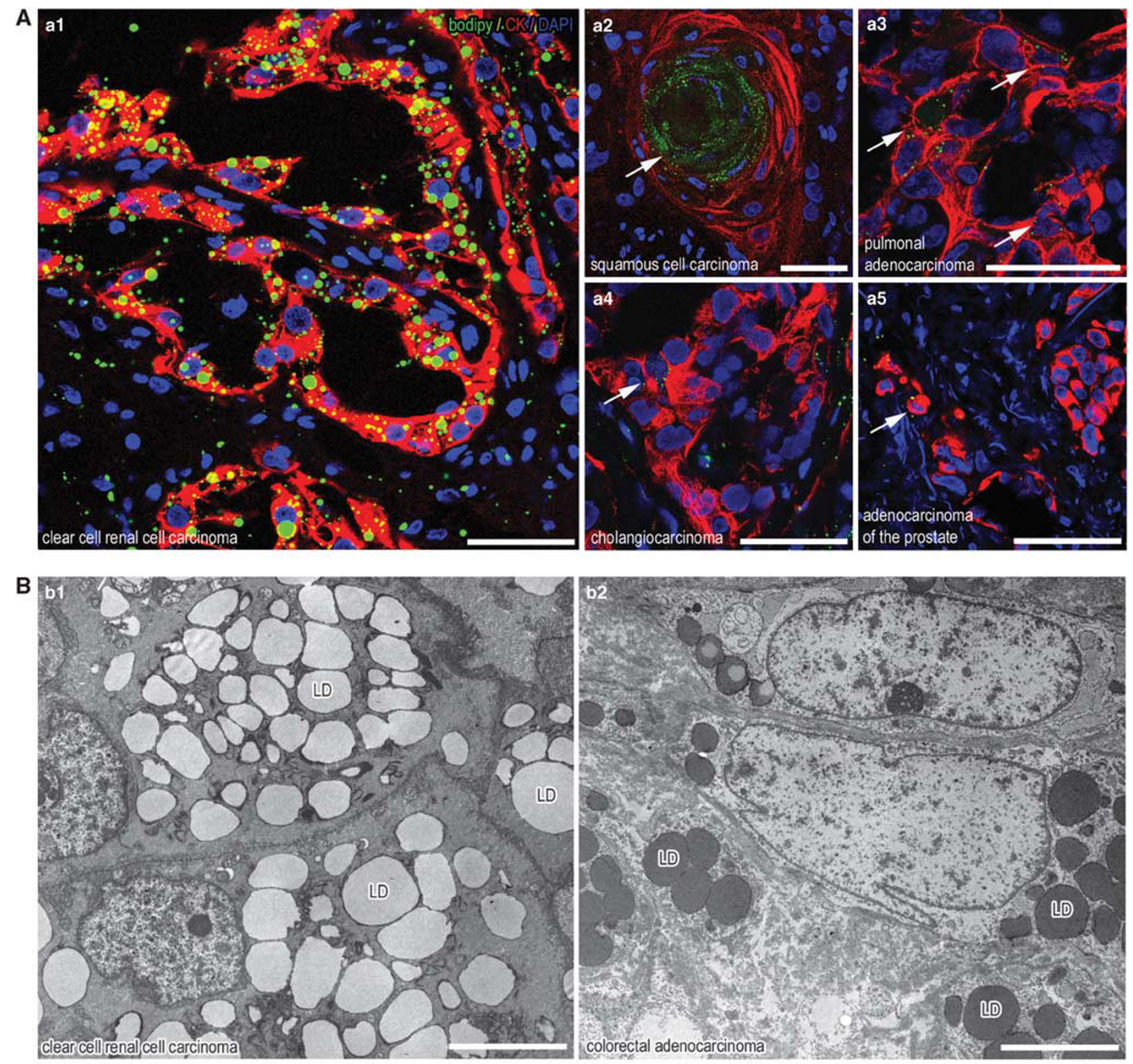

C
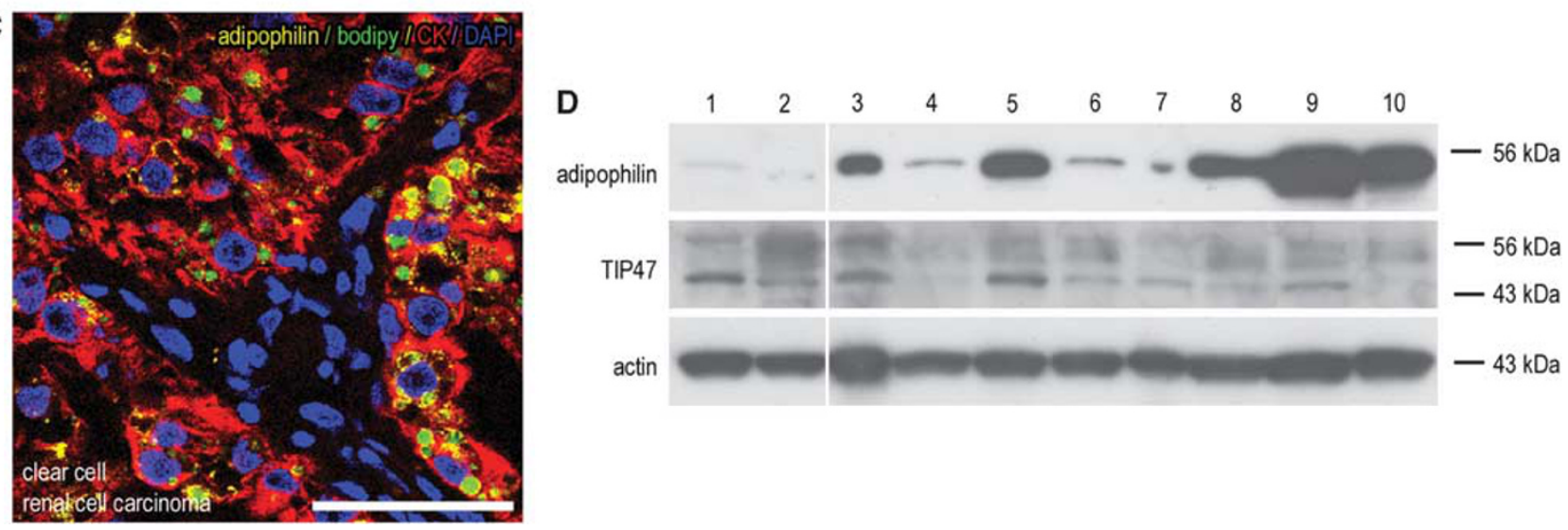
three PAT-family members, perilipin, adipophilin, and TIP47, was noted in tumors with hepatocellular (hepatocellular adenoma and carcinoma), adipocytic (liposarcoma, hibernoma, angiomyolipoma), and sebaceous differentiation (sebaceous adenoma and carcinoma; Table 1; Figure 3). Thus, PAT-expression in tumors largely reflected the expression pattern in the respective nonneoplastic tissues.

Next, we correlated PAT-expression with clinical and tumor-specific data using a large number of specimens from different carcinoma entities such as colorectal adenocarcinoma, renal cell carcinoma, tumors of the breast, lung and skin (Table 1; Supplementary Table 1). When the PAT-staining intensities in carcinomas were compared with the respective normal epithelia, adipophilin- and TIP47-positive lipid droplets were often more frequent in carcinomas as in colorectal adenocarcinoma (adipophilin, $P<0.05$; TIP47, $P<0.01$ ), carcinomas of the lung (TIP47, $P<0.01$ ), and renal cell carcinoma. In skin, mammary gland, and lung, where in particular adipophilin expression was already high in the normal epithelium due to tissue-specific functions, staining intensity in carcinomas was equal or slightly diminished. As to tumor staging and grading, in colorectal adenocarcinoma, carcinomas of the lung, and squamous cell carcinoma of the skin, TIP47 staining intensity was positively correlated with tumor size $(P<0.01)$, in colorectal adenocarcinoma and tumors of the lung additionally with tumor stage and grade $(P<0.01)$. In clear cell renal cell carcinoma TIP47 was especially high in metastases $(P<0.05)$. In carcinomas of the breast, adipophilin staining intensity in carcinomas positively correlated with cell proliferation as measured by Ki-67 staining $(P<0.05)$. In contrast, in clear cell renal cell carcinoma with especially high amount of adipophilin-positive lipid droplets, adipophilin staining intensity negatively correlated with tumor grade $(P<0.05)$. As expected, adipophilin and TIP47-positive lipid droplets were especially frequent in certain tumor types. Adipophilin-positive lipid droplets were detected in much higher amount in clear cell renal cell carcinoma than in papillary or chromophobe renal cell carcinoma $(P<0.01)$. Higher amounts of TIP47-positive lipid droplets were observed in squamous cell carcinoma and pleomorphic carcinoma in comparison to adenocarcinoma of the lung $(P<0.01)$, and in basal cell carcinoma in comparison to squamous cell carcinoma of the skin $(P<0.01)$. For all tumors analyzed, no correlation of PAT-expression was found with age and gender, and for tumors of the breast, also no correlation was found with receptor status (estrogen and progesterone receptors), nor with expression of HER2neu, p53, and BCL2.

\section{Aberrant PAT-Expression in Liver Carcinogenesis}

Because all three PAT-members can be expressed in HCC, and HCC frequently shows pronounced fatty change, we focused on expression and pattern of PAT-proteins in human HCC. By immunofluorescence microscopy, perilipin and adipophilin surrounded Nile red- or Bodipy-positive lipid droplets of different sizes in HCC cells (Figure 4). Perilipin and adipophilin showed regional heterogeneity within HCC with largely exclusive localization of adipophilin and perilipin and focal colocalization at very few lipid droplets (Figure 4). In some HCCs, perilipin, adipophilin, and especially TIP47 were only diffusely localized in the cytoplasm. Perilipin expression was variable, and if positive, heterogeneous in a subpopulation of tumor cells (see eg Figure 5; Supplementary Figure 1). Although perilipin- and adipophilin-positive lipid droplets were often oriented to the sinusoidal surface of steatotic hepatocytes in fatty liver disease, in HCC cells they were more and more randomly distributed with increasing dedifferentiation (Figure 5; Supplementary Figure 1). Only minor amounts of TIP47 in steatotic hepatocytes as well as HCC cells localized to very small lipid droplets.

To determine the frequency of PAT-proteins in human hepatocarcinogenesis, we performed immunohistology using a representative panel of human HCC and dysplastic nodules, in comparison to hepatocellular adenoma, focal nodular hyperplasia, nontumorous liver specimens mainly with cirrhosis, and normal liver specimens (Table 1b). Perilipin was detected in $30 \%$ of HCCs, whereas adipophilin and TIP47 were at least focally positive in nearly all HCCs (see Table 1b; Figure 5;

Figure 1 Accumulation of small lipid droplets is a frequent feature in human carcinomas. (a) In confocal laser scanning immunofluorescence microscopy, multiple Bodipy-positive lipid droplets (green; arrows) are present in keratin-positive carcinoma cells (red; nuclei: DAPI, blue) in clear cell renal cell carcinoma (a1), squamous cell carcinoma of the skin (a2), pulmonal adenocarcinoma (a3), cholangiocarcinoma (a4), and adenocarcinoma of the prostate gland (a5). Bars $=50 \mu \mathrm{m}$. (b) Electron microscopy shows numerous lipid droplets in clear cell renal cell carcinoma (b1) and colorectal adenocarcinoma (b2). Owing to different processing, lipid droplets are more electron dense in the colorectal adenocarcinoma in comparison to the renal cell carcinoma sample. Bars $=5 \mu \mathrm{m}$. (c) In confocal laser scanning immunofluorescence microscopy, the PAT protein adipophilin (yellow) surrounds Bodipy-positive lipid droplets (green) in cytokeratin 8/18-positive renal cell carcinoma cells (CK, red; DAPI, blue). Bar $=50 \mu \mathrm{m}$. (d) In immunoblot analyses of whole tissue lysates of different carcinomas, antibodies against adipophilin and TIP47 react with protein bands of about 53 and $47 \mathrm{kDa}$, respectively, in squamous cell carcinoma of the skin (lane 1), and of the larynx (lane 2), in adenocarcinoma of the stomach (lane 3), hepatocellular carcinoma (lane 4), cholangiocarcinoma (lane 5), ductal adenocarcinoma of the pancreas (lane 6), and of the colon (lane 7), in clear cell renal cell carcinoma (lane 8), adenocarcinoma of the prostate gland (lane 9), and ductal invasive carcinoma of the mammary gland (lane 10). Protein amounts were adjusted with actin (see below). Molecular mass markers are given on the right side. 

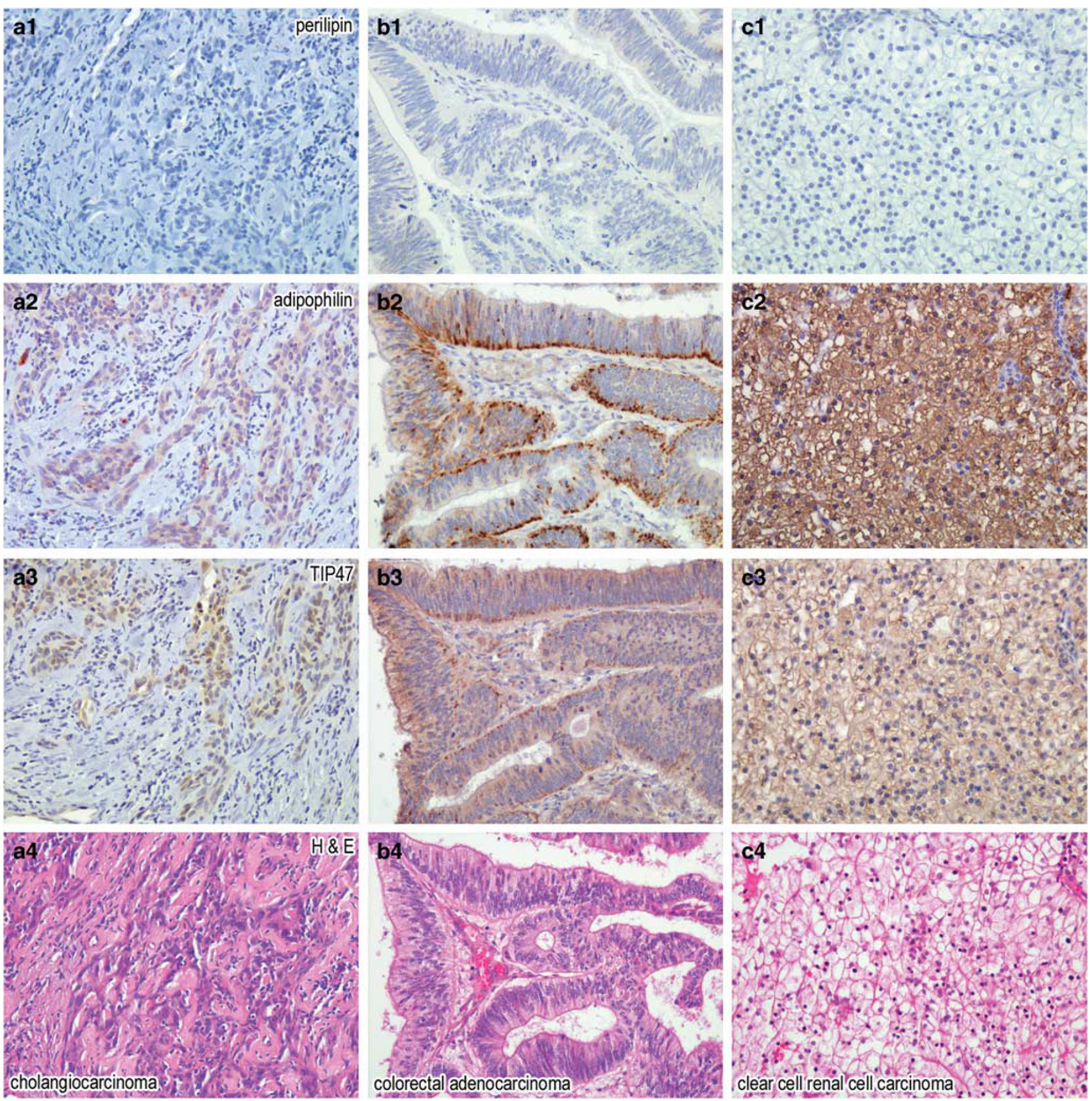

Figure 2 Examples of immunohistochemical PAT-staining in perilipin-negative human tumors. Antibodies against adipophilin (a2-c2), and TIP47 (a3-c3) react with small lipid droplets in the cytoplasm of cholangiocarcinoma (a1-a4), adenocarcinoma of the colon (b1-b4), and clear cell renal cell carcinoma (c1-c4), whereas no staining is detected with antibodies against perilipin (a1-c1). The respective H\&E stain is shown (a4-c4). Original magnification: $\times 200$.

Supplementary Figure 1). In comparison, in the respective normal (nonsteatotic) and surrounding nontumorous liver tissues (with chronic liver diseases such as steatosis, steatohepatitis, and cirrhosis), lipid droplets were positive for perilipin in $70 \%$, for adipophilin also in about $70 \%$ and for TIP47 in about $30 \%$. No significant correlations of perilipin, adipophilin, or TIP47 staining intensities with tumor etiology (eg HBV or HCV infection) or other parameters (eg gender, age, and tumor staging) were detected. Interestingly, the extent of adipophilin-positive lipid droplets positively correlated with tumor cell proliferation as determined by Ki-67 staining $(P<0.01)$. Adipophilin was upregulated already during early hepatocarcinogenesis, as surrounding nontumorous hepatocytes expressed less adipophilin than dysplastic nodules, and HCCs $(P<0.01$; Supplementary Figure 2). In contrast, 

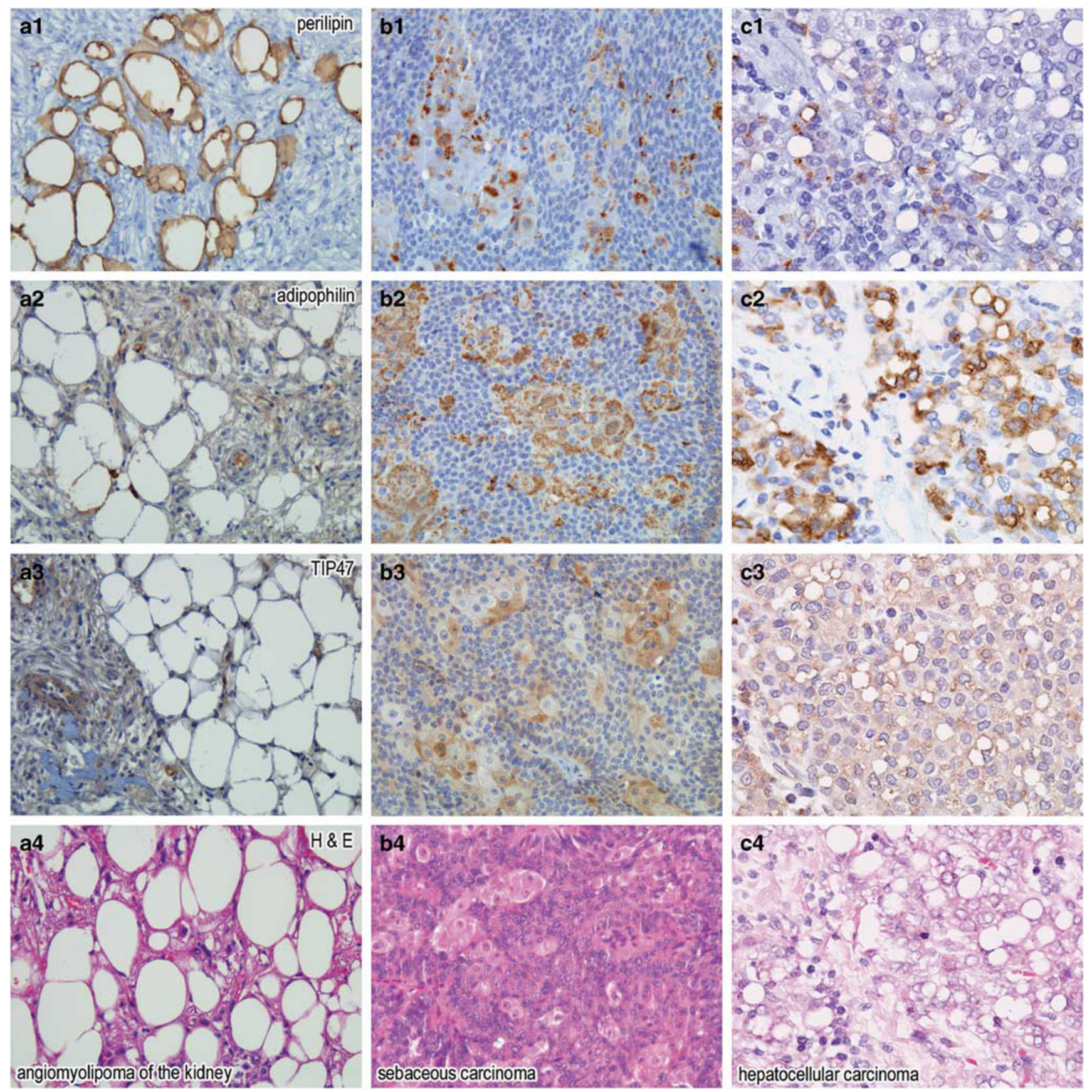

Figure 3 Examples of immunohistochemical PAT-staining in perilipin-positive human tumors. Positive staining of perilipin (a1-c1), adipophilin (a2-c2), and TIP47 (a3-c3) is detected in angiomyolipoma of the kidney (a1-a4), sebaceous carcinoma (b1-b4) and hepatocellular carcinoma (c1-c4). The respective H\&E stain is shown (a4-c4). Original magnification: $\times 200(\mathbf{a}, \mathbf{b}) ; \times 400(\mathbf{c})$.

perilipin was significantly downregulated during early hepatocarcinogenesis, as nontumorous (steatotic) hepatocytes more often expressed perilipin than dysplastic nodules and HCCs $(P<0.01$; Supplementary Figure 2). Antibodies against perilipin isoform A reacted with protein bands of about $60 \mathrm{kDa}$ in HCCs, but also in varying amounts with additional protein bands of about $55-70 \mathrm{kDa}$ (Figure 6b), probably representing protein modifications. An antibody recognizing all perilipin splice variants (clone 117.12) showed a comparable reaction pattern. In benign tumors and tumor-like lesions of the liver, such as hepatocellular adenoma and focal nodular hyperplasia, compatible with their visible fatty change, perilipin-, adipophilin-, and TIP47-positive lipid droplets were frequently detected (Table 1b) and distributed relatively even. In summary, although adipophilin is upregulated during hepatocarcinogenesis, perilipin frequently is downregulated. 

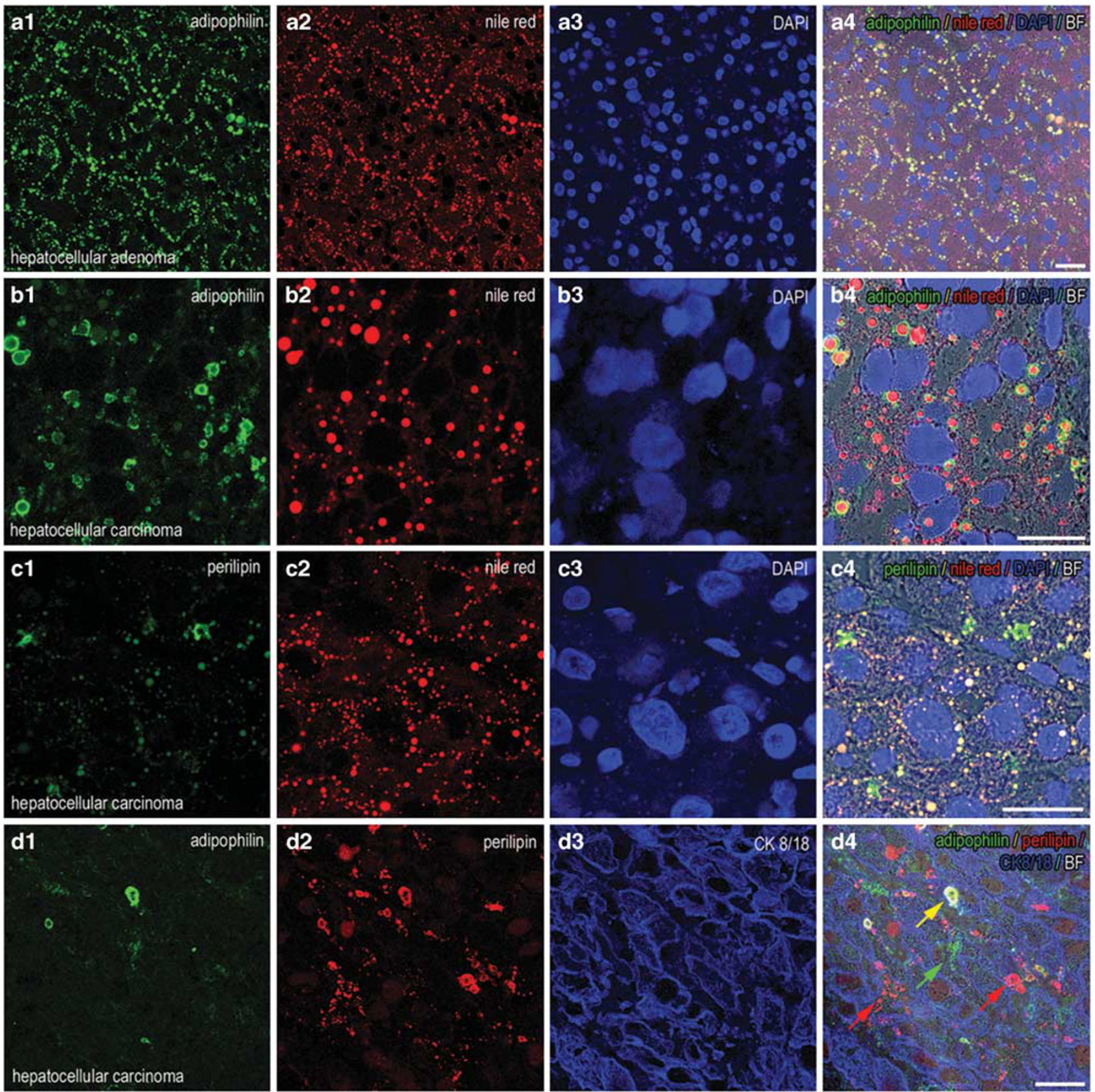

Figure 4 Perilipin and adipophilin surround lipid droplets in hepatocellular adenoma and carcinoma. Confocal laser scanning microscopy of human hepatocellular adenoma (a1-4) and carcinoma (b1-4, c1-4) shows the presence of numerous Nile red-positive lipid droplets (red, a2-c2) surrounded by adipophilin (green, a1, b1) and perilipin (green, c1). Adipophilin (green, d1) and perilipin (red, d2) are differentially expressed in cytokeratin 8/18 (CK8/18, blue, d3)-positive hepatocellular carcinoma cells with mutually exclusive localization of adipophilin (green arrow) or perilipin (red arrow) or colocalization of both (yellow arrow, d4). BF, bright field. Bars $=25 \mu \mathrm{m}$.

Because PAT-transcript expression is significantly regulated by PPARs, we next analyzed PPAR-expression in correlation with PAT-expression. Interestingly, perilipin transcripts were detectable in a higher percentage of HCCs than perilipin protein (Figure 6a and b). Also in the HCC-derived cell lines PLC/PRF-5, HuH7, Hep3B as well as in the hepatoblastoma-derived cell line HepG2, perilipin transcripts were detected with PCR analysis, but perilipin (isoform A) protein could not be detected by immunoblot and immunofluorescence microscopy. The transcription factors PPAR $\alpha$ and PPAR $\gamma$ were expressed in nearly all HCCs, and PPAR $\alpha$ mRNA correlated with adipophilin and TIP47 transcripts, whereas PPAR $\gamma$ correlated with perilipin expression. 

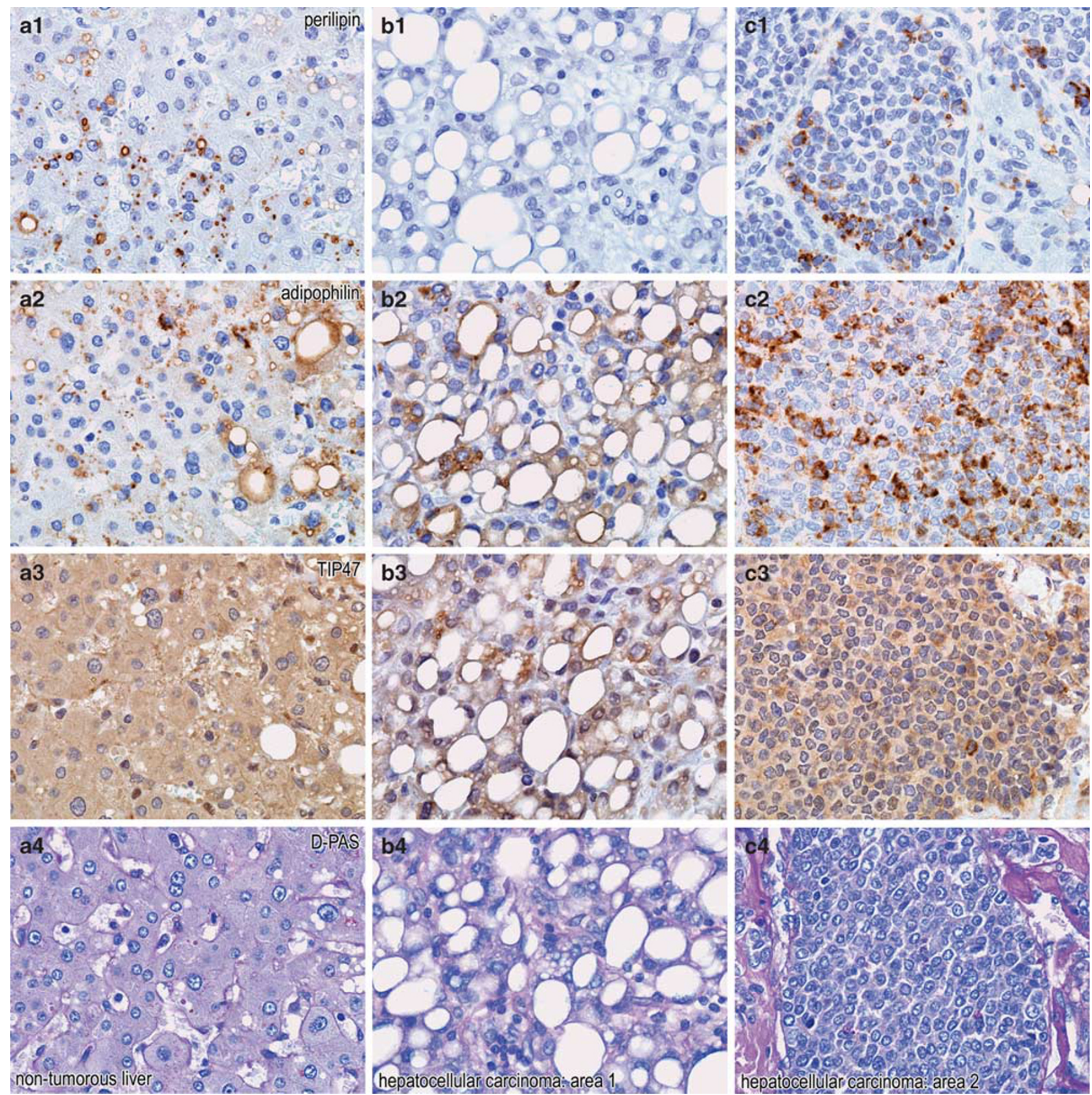

Figure 5 Regional heterogeneity of PAT expression in hepatocellular carcinoma with fatty change. Immunohistochemical analysis of perilipin (a1-c1), adipophilin (a2-c2), and TIP47 (a3-c3) in one example of a moderate to poorly differentiated human hepatocellular carcinoma with in part prominent fatty change (area 1) as well as solid areas resembling squamous cell carcinoma (area 2). Although adipophilin and TIP47 are relatively evenly distributed, perilipin reactivity is heterogenous (positivity in area 2, negativity in area 1). On the left side, the respective surrounding nontumorous liver tissue is shown (a1-a4). Diastase-PAS stain is shown (a4-c4). Original magnification: $\times 400$.

\section{Discussion}

In this study, the occurrence of lipid droplets and their correlation with lipid droplet-associated proteins of the PAT-family was investigated in a wide variety of human carcinomas by morphology, ultrastructure, and immunohistology. Extending specific analyses of single PAT-proteins in some tumor entities, ${ }^{23-25}$ our analyses show typical PAT-protein patterns in tumor cells. Whereas adipophilin and TIP47-positive lipid droplets occur in a wide variety of tumor entities, especially in carcinomas with clear cell phenotype, perilipin was only found in HCC, sebaceous tumors, and mesenchymal tumors with adipogenic differentiation. Interestingly, although carcinomas reflected PAT-expression in the respective normal tissues, the amount of adipophilin and TIP47-positive lipid droplets was often 


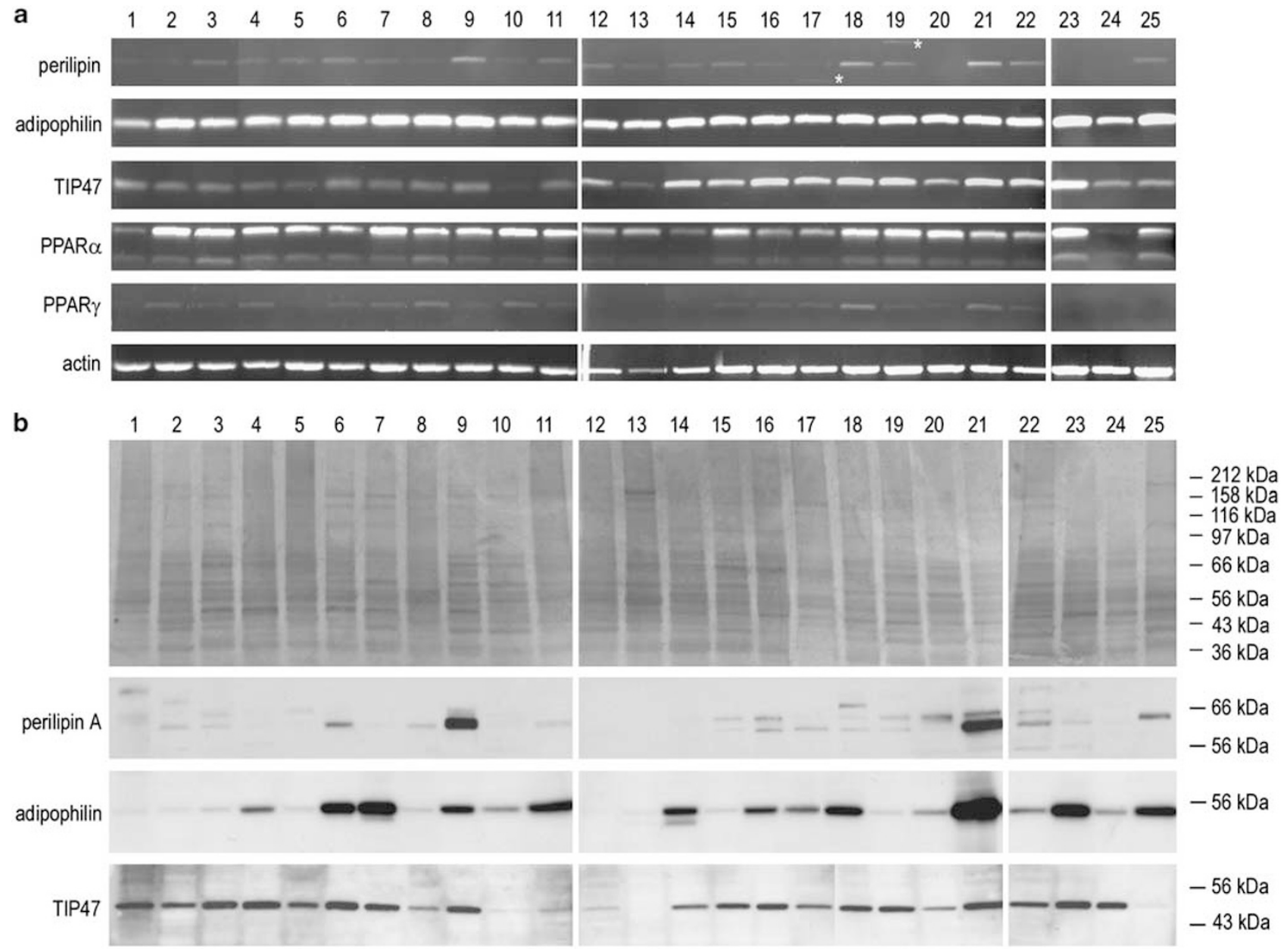

Figure 6 Perilipin, adipophilin, and TIP47 mRNA and protein expression in hepatocellular carcinoma. (a) rtPCR analysis: in 24 different human hepatocellular carcinomas (lanes 1-24) as well as in one control liver (lane 25), DNA transcripts of perilipin, adipophilin, TIP47, as well as of the transcription factors PPAR $\alpha$ and PPAR $\gamma$ were detectable at the calculated molecular weight. * In two hepatocellular carcinoma (17 and 19), one additional perilipin band was found below (17) and above (19) the estimated DNA transcripts of perilipin. rtPCR analysis was adjusted with the housekeeping gene actin. (b) Immunoblot analysis following SDS-PAGE of proteins of identical 24 hepatocellular carcinomas (1-24) and control liver (25) as shown in (a). Antibodies against adipophilin and TIP47-detected protein bands of 53 and $47 \mathrm{kDa}$, respectively, antibodies against perilipin (splice variant A) detected protein bands of about $60 \mathrm{kDa}$ as well as further protein bands between 55 and $70 \mathrm{kDa}$. Protein amounts were adjusted using Bradford assay (20 $\mu \mathrm{g}$ per lane). The respective Coomassie-blue-stained proteins are shown above. Molecular mass markers are given on the right side.

increased in carcinomas compared with the respective normal epithelia. PAT-protein pattern may actually help tissue diagnosis. Mrozinski ${ }^{25}$ has shown that perilipin positivity distinguishes liposarcoma from other sarcomas. For sebaceous carcinoma of the eyelids, perilipin, adipophilin, and TIP47 staining have been shown to visualize specifically holocrine secretion ${ }^{24,29}$ and antibodies against perilipin, adipophilin, and TIP47 are already used for the immunohistochemical diagnosis of this tumor entity. In clear cell renal cell carcinoma, adipophilin expression has been correlated with good prognosis, and transcription of adipophilin may be regulated by the von Hippel-Lindau factor/ hypoxia-inducible pathway and responsible for the typical appearance of clear cell renal cell carcinoma. ${ }^{23}$ Considering the fact that sebaceous carcinoma and lipogenic tumors pose no differential diagnostic problem to HCC, presence of perilipin may help establish hepatocellular origin in metastases of unknown origin. Recently reported broad-spectrum perilipin staining with a polyclonal rabbit antibody (Atlas Antibodies; http://www.proteinatlas.org) is not specific to perilipin, as we could show crossreaction of the purchased anti-perilipin antibody with both recombinant human perilipin and adipophilin (Supplementary Figure 3).

Another relevant finding of our study is the much higher abundance of lipid droplets regarding tumor entities and percentage of affected tumors as highlighted by PAT-protein staining and confirmed by ultrastructural analysis. For example in HCC, all three major lipid droplet-associated proteins, perilipin, adipophilin, and TIP47, outline a much higher percentage of lipid droplets (up to 90\%) than discernable by light microscopy (approximately 
$10 \% ;^{30}$ and our own analyses). No significant correlation was detectable for PAT expression regarding the etiology of HCC, a finding in accordance with Kurokawa et $a l^{31}$ who predicted using PCR array analysis that adipophilin may have a common key role in hepatocarcinogenesis. We could show that there is significant and differential dynamics in PAT-expression during step-wise tumor development as exemplified by hepatocarcinogenesis. In our study, adipophilin expression increased during hepatocarcinogenesis and in correlation with cell proliferation, whereas perilipin expression was rather reduced. As perilipin is likely to function mainly in long-term storage and release of lipid droplets, eg, in adipose tissue, ${ }^{11}$ and adipophilin and TIP47 coat smaller lipid droplets, ${ }^{32}$ which are likely to be generated and released faster, rapidly dividing tumor cells may rather require and generate adipophilin- and TIP47-type lipid droplets than perilipintype lipid droplets. Therefore, perilipin expression in adipogenic, hepatocellular, and sebaceous tumors may rather reflect the cellular differentiation of these tumor entities than a metabolic phenotype promoting tumorigenesis itself, as perilipin is often downregulated during malignant progression. The increased number of adipophilin-positive lipid droplets in HCC cells when compared with normal hepatocytes may also reflect the upregulation of lipogenic pathways as a common mechanism in different tumors. ${ }^{6-7}$ We therefore specifically addressed the question, how perilipin and adipophilin expression in liver tumors is regulated. Indeed, PPARs have major roles in the regulation of lipid metabolism. ${ }^{19}$ PPAR $\alpha$, for example, has recently been implicated in HCV-induced hepatocarcinogenesis, ${ }^{33}$ and hepatic steatosis is induced through HBx-dependent activation of $\operatorname{PPAR} \gamma$ by $\mathrm{HBV} \mathrm{X}$ protein. ${ }^{34}$ Although adipophilin has been reported to be influenced by PPAR $\alpha$, eg, in liver, perilipin is upregulated under the presence of PPAR $\gamma$ in adipocytes. Using rtPCR analysis, we detected transcripts of both PPAR $\alpha$ and PPAR $\gamma$ in human HCCs concomitant with perilipin, adipophilin, and TIP47 expression, suggesting PPARs as potential regulators of altered PAT-expression in neoplastic steatogenesis. Apart from PPARs, also other pathways have been shown to modulate lipogenesis in cancer cells, ie, growth factor signaling through PI3K/Akt pathway or steroid hormone signaling, which lead to activation of SREBP-1. ${ }^{7}$ Recent studies indicate that lipid droplets and associated proteins may also be significantly regulated on the posttranscriptional level, eg, through ubiquitinylation, ${ }^{35,36}$ a process known to be aberrant in malignant tumors. ${ }^{37}$

In conclusion, PAT-proteins, especially perilipin, show differential expression regarding tumor entities and may support differential diagnosis. Furthermore, PAT-proteins show that lipid droplet formation is a very common phenomenon in cancer cells, much more frequent than expected by conventional light microscopy. Thus, interference with
PAT-proteins such as adipophilin or TIP47 may have substantial metabolic effects on tumor cells or even antineoplastic potential, as shown for the inhibition of fatty acid synthase. ${ }^{21-22}$

\section{Acknowledgements}

We thank Elisabeth Specht-Delius, Maria König, Lena Pawella, Sabine Fraschka, Michaela Bissinger, Bettina Walter, Heide Schumacher, Zlata Antoni, Csaba Toth, and Annett Müller for technical help, as well as Barbara Schreiber for assistance in the documentation of the TMAs. We also thank Jens Schumacher as well as Thomas W Keenan for critically reading the paper and Werner W Franke for encouragement throughout the study. BKS was a member of the postdoc program and is now supported by a stipend from the Olympia-Morata program of the Medical Faculty of Heidelberg University.

\section{Disclosure/conflict of interest}

The authors declare no conflict of interest.

\section{References}

1 Warburg O. On the origin of cancer cells. Science 1956;123:309-314.

2 Vander Heiden MG, Cantley LC, Thompson CB. Understanding the Warburg effect: the metabolic requirements of cell proliferation. Science 2009;324: 1029-1033.

3 Menendez JA, Lupu R. Fatty acid synthase and the lipogenic phenotype in cancer pathogenesis. Nat Rev Cancer 2007;7:763-777.

4 Yahagi N, Shimano H, Hasegawa K, et al. Co-ordinate activation of lipogenic enzymes in hepatocellular carcinoma. Eur J Cancer 2005;41:1316-1322.

5 Tennant DA, Durán RV, Boulahbel H, et al. Metabolic transformation in cancer. Carcinogenesis 2009;30: 1269-1280.

6 Swinnen JV, Brusselmans K, Verhoeven G. Increased lipogenesis in cancer cells: new players, novel targets. Curr Opin Clin Nutr Metab Care 2006;9:358-365.

7 Yamashita T, Honda M, Takatori H, et al. Activation of lipogenic pathway correlates with cell proliferation and poor prognosis in hepatocellular carcinoma. J Hepatol 2009;50:100-110.

8 Kutami R, Nakashima Y, Nakashima O, et al. Pathomorphologic study on the mechanism of fatty change in small hepatocellular carcinoma of humans. J Hepatol 2000;33:282-289.

9 International Consensus Group for Hepatocellular Neoplasia. Pathologic diagnosis of early hepatocellular carcinoma: a report of the International Consensus Group for Hepatocellular Neoplasia. Hepatology 2009;49:658-664.

10 Fujimoto T, Ohsaki Y, Cheng J, et al. Lipid droplets: a classic organelle with new outfits. Histochem Cell Biol 2008;130:263-279. 
11 Greenberg AS, Egan JJ, Wek SA, et al. Perilipin, a major hormonally regulated adipocyte-specific phosphoprotein associated with the periphery of lipid storage droplets. J Biol Chem 1991;266:11341-11346.

12 Brasaemle DL, Barber T, Wolins NE, et al. Adipose differentiation-related protein is an ubiquitously expressed lipid storage droplet-associated protein. J Lipid Res 1997;38:2249-2263.

13 Heid HW, Moll R, Schwetlick I, et al. Adipophilin is a specific marker of lipid accumulation in diverse cell types and diseases. Cell Tissue Res 1998;294:309-321.

14 Wolins NE, Rubin B, Brasaemle DL. TIP47 associates with lipid droplets. J Biol Chem 2001;276:5101-5108.

15 Kimmel AR, Brasaemle DL, McAndrews-Hill M, et al. Adoption of PERILIPIN as a unifying nomenclature for the mammalian PAT-family of intracellular, lipid storage droplet proteins. J Lipid Res 2009; e-pub ahead of print.

16 Martinez-Botas J, Anderson JB, Tessier D, et al. Absence of perilipin results in leanness and reverses obesity in Lepr(db/db) mice. Nat Genet 2000;26: 474-479.

17 Tansey JT, Sztalryd C, Gruia-Gray J, et al. Perilipin ablation results in a lean mouse with aberrant adipocyte lipolysis, enhanced leptin production, and resistance to diet-induced obesity. Proc Natl Acad Sci USA 2001;98:6494-6499.

18 Chang $\mathrm{BH}$, Li L, Paul A, et al. Protection against fatty liver but normal adipogenesis in mice lacking adipose differentiation-related protein. Mol Cell Biol 2006;26: 1063-1076.

19 Shimizu M, Yamashita D, Yamaguchi T, et al. Aspects of the regulatory mechanisms of PPAR functions: analysis of a bidirectional response element and regulation by sumoylation. Mol Cell Biochem 2006;286:33-42.

20 Straub BK, Stoeffel P, Heid H, et al. Differential pattern of lipid droplet-associated proteins and de novo perilipin expression in hepatocyte steatogenesis. Hepatology 2008;47:1936-1946.

21 Kuhajda FP, Jenner K, Wood FD, et al. Fatty acid synthesis: a potential selective target for antineoplastic therapy. Proc Natl Acad Sci USA 1994;91:6379-6383.

22 Kuhajda FP. Fatty-acid synthase and human cancer: new perspectives on its role in tumour biology. Nutrition 2000;16:202-208.

23 Yao M, Tabuchi H, Nagashima Y, et al. Gene expression analysis of renal carcinoma: adipose differentiationrelated protein as a potential diagnostic and prognostic biomarker for clear-cell renal carcinoma. J Pathol 2005;205:377-387.
24 Muthusamy K, Halbert G, Roberts F. Immunohistochemical staining for adipophilin, perilipin and TIP47. J Clin Pathol 2006;59:1166-1170.

25 Mrozinski J. Immunhistochemische Identifikation und Klassifizierung von Liposarkomen mittels verschiedener Fett-assoziierter Proteine. Medical Dissertation. Philipps-University Marburg: Germany, 2007;1-103.

26 Remmele W, Stegner HE. [Recommendation for uniform definition of an immunoreactive score (IRS) for immunohistochemical estrogen receptor detection (ER-ICA) in breast cancer tissue]. Pathologe 1987;8: 138-140.

27 Brunt EM, Janney CG, Di Bisceglie AM, et al. Nonalcoholic steatohepatitis: a proposal for grading and staging the histological lesions. Am J Gastroenterol 1999;94:2467-2474.

28 Singer S, Ehemann V, Brauckhoff A, et al. Protumourigenic overexpression of stathmin/Op18 by gain-of-function mutation in p53 in human hepatocarcinogenesis. Hepatology 2007;46:759-768.

29 Izumi M, Mukai K, Nagai T, et al. Sebaceous carcinoma of the eyelids: thirty cases from Japan. Pathol Int 2008;58:483-488.

30 Goodman ZD, Terraciano LM. Tumours and tumourlike lesions of the liver. MacSween's Pathology of the Liver 5th edn. Churchill Livingstone, Elsevier: Philadelphia, 2007;761-814.

31 Kurokawa Y, Matoba R, Nakamori S, et al. PCR-array gene expression profiling of hepatocellular carcinoma. J Exp Clin Cancer Ress 2004;23:135-141.

32 Wolins NE, Brasaemle DL, Bickel PE. A proposed model of fat packaging by exchangeable lipid droplet proteins. FEBS Lett 2006;580:5484-5491.

33 Tanaka N, Moriya K, Kiyosawa K, et al. PPARalpha activation is essential for HCV core protein-induced hepatic steatosis and hepatocellular carcinoma in mice. J Clin Invest 2008;118:683-694.

$34 \mathrm{Kim} \mathrm{KH}$, Shin HJ, Kim K, et al. Hepatitis B virus X protein induces hepatic steatosis via transcriptional activation of SREBP1 and PPARgamma. Gastroenterology 2007;132:1955-1967.

35 Eastman SW, Yassaee M, Bieniasz PD. A role for ubiquitin ligases and Spartin/SPG20 in lipid droplet turnover. J Cell Biol 2009;184:881-894.

36 Hall AM, Brunt EM, Chen Z, et al. Dynamic and differential regulation of proteins that coat lipid droplets in fatty liver dystrophic mice. J Lipid Res 2009; e-pub ahead of print.

37 Mani A, Gelmann EP. The ubiquitin-proteasome pathway and its role in cancer. J Clin Oncol 2005;23: 4776-4789.

Supplementary Information accompanies the paper on Modern Pathology website (http://www.nature.com/ modpathol) 